\title{
Der Homunkulus und die Zeit. Warum die Neurophysiologie die Frage des freien Willens nicht lösen kann
}

\section{Giovanni Galizia}

Zur Frage nach der Evolution der Freiheit und nach der Funktion des Bewusstseins berichte ich aus dem Blickwinkel meiner eigenen Disziplin, nämlich den Neurowissenschaften. ${ }^{1}$ Ich werde dabei auf vier Aspekte eingehen:

1. Die Rolle einzelner Disziplinen im interdisziplinären Diskurs.

2. Das Ausmaß der Kompetenz und die Grenzen der Neurowissenschaften.

3. Die Frage, wie Tiere Entscheidungen treffen. ${ }^{2}$

1 Auch in den Neurowissenschaften - wie in jeder anderen Disziplin - werden durchaus unterschiedliche Positionen bezogen. Meine Stellungnahme kommt aus den Neurowissenschaften, aber ich spreche nicht für alle Neurowissęnschaftler.

2 In jedem interdisziplinären Diskurs kann die Wahl eines Wortes zu Missverständnissen, manchmal zu Abwehrreaktionen und gelegentlich zu direkter Ablehnung eines Gedankenganges führen, ohne dass diese Reaktion durch den Inhalt der Abhandlung begründet wäre. Ein solches Wort ist "Entscheidung“. Für viele ist „Entscheidung" ein intentionaler Akt und setzt damit Bewusstsein voraus. Der Satz ,ein Tier entscheidet sich“ würde also voraussetzen, dass ein Tier Bewusstsein hat. Ein Leser, der a priori dem Tier Bewusstsein abstreitet, würde ab dieser Stelle den Text nicht mehr wohlwollend, sondern ablehnend lesen. Ein Leser, der die Bewusstseinsfrage offen lässt, würde ab dieser Stelle jede Argumentation über Bewusstsein für zirkulär halten, da die Wortwahl schon eine Stellungnahme impliziert. Leider habe ich aber kein Wort gefunden, das nicht entweder für manche ein Bewusstsein impliziert oder gerade ein Bewusstsein ausschließt (etwa "Reaktionsmechanismus"). Hinzu kommt, dass innerhalb der Neurowissenschaften „Entscheidung“ ausdrücklich nicht Intentionalität impliziert. Bei Menschen kann man von „,bewusster Entscheidung“ und ,unbewusster Entscheidung" sprechen oder sich bei Entscheidungen fragen, ob sie intentional waren oder nicht (was nicht ginge, wenn das Wort per se schon die Intentionalität voraussetzen würde). Bei Tieren können wir beobachten, dass in einer Verhaltenssituation, in der mehrere Verhaltensweisen (sagen wir A, B und C) möglich sind, ein Tier eine Verhaltensweise ausführt es hat sich fuir ein Verhalten entschieden. Wir sagen „das Tier hat sich für A 
4. Die Tatsache, dass „Bewusstsein“" weder zeitlich noch räumlich zu verorten ist.

Letzteres hat unter anderem zur Folge, dass unsere umgangssprachliche Verwendung des Begriffs „Bewusstsein“ in den Neurowissenschaften nicht brauchbar ist. Der Begriff "Bewusstsein“ und auch der Begriff „Freiheit" sind neurobiologisch nicht so definierbar, wie sie in der Philosophie verwendet werden. Auch hier herrscht jedoch keine Einigkeit über den angemessenen Begriffsgebrauch. Um Missverständnisse zu vermeiden, sollten diese Begriffe in den Neurowissenschaften mit großer Vorsicht benutzt werden.

\section{Die Rolle einzelner Disziplinen im interdisziplinären Diskurs}

Im Antrag für den vom BMBF geförderten Forscherverbund „Funktionen des Bewusstseins ${ }^{\text {“3 }}$ findet sich folgender Satz: „In Bezug auf hervorragende Interdisziplinarität, die eine Chance hat, die mit Sicherheit auftretenden Schwierigkeiten der terminologischen Verständigung über die Fachgrenzen hinaus zu meistern, sind wir der Überzeugung, dass die erste Voraussetzung der beteiligten Wissenschaftler disziplinäre Exzellenz sein muss. Nur mit hinreichender fachlicher Kompetenz innerhalb der einzelnen Disziplin stellt sich die nötige Souveränität ein, die Terminologie der eigenen Disziplin zu hinterfragen und für neue Einflüsse zu öffnen, ohne dabei die Verbindung zur ursprünglichen Ausgangsdisziplin zu verlieren." (Gerhardt/Heilinger, 2005) An den diesem Sammelband zugrunde liegenden Treffen beteiligten sich Philosophen, Psychologen, Psychiater, Neurowissenschaftler, Biologen - eine weit gefasste Gruppe, und noch andere Disziplinen könnten mitreden, etwa Theologen, Mystiker oder auch nachdenkliche

entschieden" (,the animal has decided for A“ - das Fehlen des reflexiven „sich" im Englischen macht diese Wortwahl im angelsächsischen Sprachraum weniger kompromittierend). Die Frage nach dem Bewusstsein und der Freiheit ist innerhalb der neurowissenschaftlichen Sprachwahl vom Wort „Entscheidung" entkoppelt. In diesem Sinne verwende ich in meinem Text „Entscheidung und vertraue auf die geistige Flexibilität bei den Lesern mit anderen disziplinären Wurzeln.

3 Die Forschergruppe Funktionen des Bet»usstseins arbeitet seit 2006 an der BerlinBrandenburgischen Akademie der Wissenschaften. 
Nichtintellektuelle. Diese Vielfalt hat Konsequenzen für den Diskurs, der zwischen den Beteiligten stattfindet.

Wir denken über das Gleiche nach: „Bewusstsein“. Aber wir sprechen andere Sprachen, denn unsere Wissenschaften haben andere Fragestellungen, gehen von anderen Grundlagen aus und bauen auf unterschiedlichen Methoden auf. Darum ist es gerade im interdisziplinären Diskurs nötig, die Voraussetzungen jeder Disziplin klar zu benennen. Ich bin kein Philosoph, wenngleich ich gerne philosophischen Beiträgen lausche und aus meiner Laienposition heraus mitphilosophiere. Trotzdem ist mir klar, dass ich die philosophischen Beiträge nicht mit der Tiefe durchdringen kann, die jemand hat, der oder die über Jahre hinweg philosophisch geschult wurde. Ich denke, dass dies auch in der anderen Richtung gilt: Wir stoßen im interdisziplinären Diskurs an Grenzen. ${ }^{4}$ Hiermit meine ich nicht die intellektuellen Grenzen der Beteiligten. Wohl sind es zum Teil Grenzen, die durch ungleiches Wissen entstehen. Vor allem aber sind es konzeptionelle und methodische Grenzen, die tiefer greifen. Die Philosophie ist eine nachdenkliche Wissenschaft, auch dann, wenn sie sich an der Realität orientiert. Die Neurowissenschaften sind eine rein empirische Wissenschaft, auch wenn wir gelegentlich nachdenken. In dem Moment, in dem wir uns auf der Basis unseres neurowissenschaftlichen Wissens ein Bild über philosophische Fragen machen, verlassen wir aber den Boden der Neurowissenschaften. Ich sage das nicht negativ wertend, im Gegenteil: Ich denke, dass gerade diese Grenzüberschreitungen notwendig sind und das Potenzial bieten, Neuland zu betreten. Aber ich sage es einschränkend: Es muss klar sein, dass Erkenntnisse, die durch solche Exkurse gewonnen werden, nicht aus der Neurobiologie kommen. Wenn also, um ein Beispiel zu nennen, die Willensfreiheit von einem Neurobiologen negiert wird, so geschieht das nicht in seiner Funktion als Neurobiologe, sondern als Philosoph, der auf der Basis seiner neu-

4 Die oben erwähnte Problematik bei der Wortwahl („Entscheidungen“) ist ein gutes Beispiel. Hier müssen wir beim Zuhören die Definition der anderen Disziplin akzeptieren, beim Sprechen sollten wir aber die eigene Definition benutzen und nicht versuchen, eine uns fremde zu nehmen. Im interdisziplinären Diskurs ist die Flexibilität auf der Seite der Zuhörer gefragt. Darin unterscheidet sich der interdisziplinäre Diskurs grundsätzlich von der Popularisierung der Wissenschaften: Bei allgemeinverständlichen Vorträgen muss die Flexibilität auf der Seite des Redners gegeben sein. 
robiologischen Kenntnisse argumentiert. ${ }^{5}$ Vielleicht kann man ihn einen Neurophilosophen nennen. Um solche Grenzziehungen treffen zu können, müssen die Grenzen klar definiert sein.

\section{Wo sind die Kompetenzen, wo die Grenzen der Neurowissenschaften?}

Die Neurowissenschaften sind eine rein empirische Wissenschaft: Nur das Experiment und die Reproduzierbarkeit haben Autorität, nur an dieser empirischen Beobachtung lässt sich die Qualität neurowissenschaftlicher Aussagen messen. Jede gebildete Theorie ist nur so gut, wie sie mit experimentellen Daten, die nach der Theoriebildung erhoben wurden, kompatibel ist. Ziel der Neurowissenschaften ist es, die Funktionsweise des Gehirns zu verstehen. Wir sind eingebettet in den Naturwissenschaften, und alles was wir machen, muss nach „unten“ kompatibel sein - „unten“ in Anführungsstrichen, denn damit ist keine Hierarchie, aber wohl eine Inklusivität gemeint. Keines unserer Modelle darf zur Biochemie im Widerspruch stehen, so wie nichts aus der Biochemie im Widerspruch zur Chemie sein darf und diese wiederum nicht im Widerspruch zur Physik. Dies ist die Neurowissenschaft, die ich hier vertrete. Die Inklusivität der Naturwissenschaften heißt aber nicht, dass der Physiker der bessere Neurowissenschaftler ist. Aus dem Anspruch auf Widerspruchsfreiheit mit der Physik entsteht kein Anspruch der Physik auf Vereinnahmung der Neurowissenschaften.

Wofür sind die Neurowissenschaften nun die ,unteren “ Disziplinen? Das sind zum Beispiel die Psychologie und die Soziologie. Ich denke, die Neurowissenschaften sollten sich in Bescheidenheit üben: Wir können nicht die Soziologie ersetzen, selbst wenn wir verlangen können, dass Befunde aus der Soziologie nicht im Widerspruch zu den neurobiologischen Grundlagen sein dürfen. Und wir müssen an das

5 Dieses Beispiel hinkt in dieser verkürzten Form. Gäbe es eine aus neurobiologischer Sicht eindeutige Definition von "Willensfreiheit", dann wäre die Aussage falsch, denn die Neurobiologie könnte innerhalb ihrer Disziplin über die Willensfreiheit forschen. Kann es aber keine neurobiologische Definition geben (weil jede Definition nur tautologisch möglich wäre), so ergibt sich die obige Aussage. Ich benutze diese hier also als Vorgriff auf den nächsten $\mathrm{Ab}$ schnitt, in dem ich argumentiere, dass es für die „Willensfreiheit" eben keine Definition aus der Neurobiologie geben kann, da diese methodisch an eine statistische Analyse gebunden ist. 
Selbstbewusstsein der anderen Disziplinen appellieren, denn oft sind es gar nicht die Neurowissenschaftler, die sich aufdrängen, sondern z.B. Journalisten und Hobbywissenschaftler, die meinen, dass alles Menschliche mit der Gehirnforschung erklärbar sein muss.

Um ein Beispiel zu nennen: Immer wieder kommt es vor, dass aus sportlichen, religiösen oder politischen Anlässen heraus Menschenmengen eine große zerstörerische Kraft entwickeln. Viele würden den einzelnen Menschen in einer solchen Menge den freien Willen absprechen. Kann ich das neurobiologisch begründen? Natürlich sind in jedem dieser Gehirne bestimmte Areale aktiv, Botenstoffe und Hormone werden ausgeschüttet, Schwellwerte in einzelnen Zellen sind so eingestellt, dass dieses Verhalten entsteht - aber genauso gut könnte ich sagen, dass bestimmte Wasserstofforbitale mit bestimmten Sauerstofforbitalen interagieren. Auf dieser Betrachtungsebene können wir den Kern des Geschehens nicht auf befriedigende Weise beschreiben und untersuchen. Um zu verstehen, was in Amok laufenden Menschenmassen passiert, ist die geschichtswissenschaftliche Sprache und die Sprache der Soziologen der Analyse von Neurowissenschaftlern überlegen. ${ }^{6}$

Die Grundfesten der Neurowissenschaften liegen in der Empirie. "Nihil est in iritellectu quod non fuerit prius in sensu" lautet der Grundsatz des Sensualismus, einer Form des Empirismus. Hinzu kommt, dass ein Befund in dieser Hinsicht nur dann glaubwürdig ist, wenn er wiederholt werden kann. Die Statistik ist unser wichtigstes Werkzeug: Wir untersuchen nur Phänomene, die zuverlässig reproduzierbar sind. Was nicht statistisch signifikant ist, fällt aus unserem Horizont heraus. Was nicht statistisch signifikant belegbar ist, darüber

6 Was für eine Erklärung kann aus den verschiedenen Disziplinen kommen? Die Soziologie könnte zum Beispiel verstehen, unter welchen Bedingungen in einer Menschenmasse Gewaltbereitschaft entsteht. Dies ist eine Ebene, auf der wir durch die gewonnenen Erkenntnisse solche Bedingungen gezielt vermeiden können (oder auch Konfliktanschürer solche Bedingungen erzeugen können, um gezielt Gewalt zu erreichen). Die nächste Ebene - die Psychologie des Einzelnen - ist schon schwieriger. Auf neurobiologischer Ebene könnte die Erkenntnis darin liegen, dass ein bestimmter Botenstoff A, wenn er in Areal X ausgeschüttet wird, die Gewaltbereitschaft erhöht, und man könnte die physiologischen Bedingungen erforschen, unter denen dies passiert. Und man will wohl wissen, welche Wasserstoffbrückenbindungen dabei gebildet werden, wenn man noch weiter die Disziplinkette verfolgt. Wohl sind die verschiedenen Erklärungsebenen gleich „wahr", und doch ist für das Verständnis der Situation die soziologische Erklärung in Allgemeinen die nützlichere. 
können wir nichts aussagen: Uns ist Wittgensteins Satz in die Seele geschrieben: „Wovon man nicht sprechen kann, darüber muss man schweigen" (Wittgenstein, 1963, 115) - dies beziehen wir gerne auf uns Naturwissenschaftler. Dies gilt für alle experimentellen Wissenschaften. Wir können den Zufall mathematisch beschreiben, und wenn wir einen Versuch machen, dann beurteilen wir das Ergebnis durch einen Vergleich mit dem errechneten Zufallsergebnis. Dadurch wissen wir, mit welcher Wahrscheinlichkeit das Versuchsergebnis ein Zufallsergebnis ist und mit welcher (komplementären) Wahrscheinlichkeit es nicht zufällig ist. $\mathrm{Um}$ das machen zu können, müssen wir den Versuch öfter wiederholen.

Das hat eine ganz grundlegende Konsequenz: Die Einmaligkeit entzieht sich dem Forschungsgegenstand einer empirischen Wissenschaft. Wir können Michelangelo nicht darum bitten, die Sixtinische Kappelle ein zweites Mal auszumalen, um zu wissen, ob er sie auch anders hätte ausmalen könmen. Und auch Beethovens Entscheidung, einen Text von Schiller in einer Symphonie zu vertonen, ist nicht wiederholbar. Wir können grundsätzlich nicht testen, ob es sich um eine freie oder eine nicht-freie, ob es sich um eine bewusste oder eine nicht-bewusste Entscheidung gehandelt hat. Dies gilt sowohl für die Entscheidungsfreiheit einzelner Menschen als auch für die Entscheidungsfreiheit ganzer Gruppen: Die Entscheidung, diese Musik zur Europäischen Hymne zu machen, ist nicht unter gleichen Bedingungen reproduzierbar. ${ }^{7}$

Das heißt natürlich nicht, dass solche Phänomene nicht wissenschaftlich untersuchbar sind - wie Phantasie sich entwickelt und unter welchen Bedingungen sie gedeiht, ist ein wichtiger Forschungsgegenstand der Psychologie. Viel Forschungsenergie befasst sich genau mit dieser Problematik: Wie lassen sich aufschlussreiche Experimente gestalten, die einmalige und nicht wiederholbare Ereignisse (wie etwa die Phantasie, die Individualität einer Person oder eine freie Entscheidung) in einen statistisch analysierbaren, methodisch reproduzierbaren Rah-

7 Mit diesen Ausfihhrungen stehe ich in meiner Disziplin nicht alleine da. Im Rahmen der Diskussionen im Humanprojekt hat Peter Hammerstein in seinem Beitrag gesagt: „In der Biologie wird nicht über Freiheit geredet", Andreas Elepfandt hat das in der Diskussion präzisiert: „Freiheit ist nicht das Suchobjekt der Biologie, weil hier die Regelmäßigkeit aufhört." Ferdinand Hucho hat diese These folgendermaßen auf den Punkt gebracht: „Freiheit ist kein biologischer Begriff". Vgl. dazu den Beitrag von Ferdinand Hucho in diesem Band. 
men zwängen. ${ }^{8}$ Während sich also die einzelne freie Entscheidung grundsätzlich der naturwissenschaftlichen Erklärung entzieht, so bleiben die Mechanismen, die eine freie Entscheidung ermöglichen, durchaus einer der wichtigsten Gegenstände auch der Neurobiologie, Das ist ein feiner, aber wichtiger Unterschied. Auf diesem Gebiet stehen uns noch große Herausforderungen bevor.

\section{Was also kann die Neurowissenschaft? Wie Tiere Entscheidungen treffen}

Ich arbeite an Insekten und will deswegen einige Beispiele aus der Welt der Bienen vorstellen. Bienen treffen tagtäglich Entscheidungen: Welche Blüte wird angeflogen, welcher Heimweg wird geflogen. Soll eine Biene lieber im Stock dem Hausdienst frönen und die Brut pflegen, oder soll sie lieber hinaus in die Welt und Pollen oder Nektar sammeln? Jede Biene muss sich einzeln entscheiden. ${ }^{9} \mathrm{Ob}$ Bienen Bewusstsein haben, wissen wir nicht, denn es gibt kein anerkanntes Experiment, das uns eine Aussage darüber erlauben würde, ob Tiere Bewusstsein haben - dazu ist die Definition von „Bewusstsein“ nicht eindeutig genug. Auch wenn wir nicht wissen können, ob Bienen Bewusstsein haben, können wir dazu eine Meinung haben, und ich denke, dass die meisten

8 Wir können durchaus untersuchen, wie viel Entscheidungsfreiheit in einem Bild von Michelangelo oder in einem Bild von Jackson Pollock ist. So sind kürzlich Verfahren entwickelt worden, die einen „echten“ von einem „falschen“" Pollock unterscheiden können: Bei einem ,echten“ Pollock folgen die Muster einer fraktalen Geometrie, während man bei einem falsehen Pollock diese Regelmäßigkeit nicht findet. (Dieser Befund ist jedoch kürzlich wieder in Frage gestellt worden.) Ist die Kontrolle, die Pollock offensichtlich über flieBende Farbe ausübte, für uns eine nützliche Information, wenn wir über sein Bewusstsein oder seinen freien Willen reden? Ich denke: nein. Dies ,ich denke" basiert aber nicht auf meiner Kompetenz als Neurowissenschaftler, denn es ist grundsätzlich sinnlos, für diese Frage auf kompetente Antworten aus den Neurowissenschaften zu hoffen.

9 Über die Schwierigkeit mit dem Begriff "Entscheidung" habe ich oben berichtet. Hier wird „Entscheidung " sehr allgemein eingesetzt und beschreibt Verhaltenssituationen, in denen verschiedene Verhaltensmöglichkeiten bestehen, von denen ein Tier eine realisiert. Was zur Ausprägung des einen und nicht des anderen Verhaltens führt, ist genau der Gegenstand der Untersuchung: angeboren, reflexiv, erlernt, abwägend, aus Gründen heraus - welche Faktoren zum Tragen kommen, soll durch die Wortwahl „Entscheidung" nicht vorweg genommen werden. 
erwachsenen Menschen meine Meinung teilen, nämlich dass Bienen kein Bewusstsein haben. ${ }^{10}$ Und trotzdem: Bienen treffen Entscheidungen, und alles, was wir in der Neurobiologie lernen, spricht dafür, dass dabei im Gehirn die gleichen Mechanismen wirken wie bei uns Menschen, wenn wir Entscheidungen treffen. Das Erforschen der Entscheidungsfindung bei Bienen kann uns also helfen, Entscheidungen von Menschen besser zu verstehen.

Entscheidungen können auf angeborenem Wissen basieren (etwa dass Bienen auf Blüten und nicht auf Steinen Nektar suchen) oder auf erlerntem Wissen (etwa dass derzeit die Lindenblüten Nektar haben). Nehmen wir das Beispiel einer erlernten Entscheidung, nämlich die, auf eine bestimmte Blüte zu fliegen. Bienen sind blütenstet: Der Imker beobachtet seinen Bienenstock, und wenn er sieht, dass die Bienen gerade die Linden entdeckt haben, dann schleudert er schnell den alten Honig aus und produziert reinen Lindenblütenhonig, denn jetzt werden alle Bienen im Stock mit Vorliebe Linden ernten. Jede Biene einzeln entscheidet sich jetzt für Lindenblüten. Diese Entscheidung führt zu weiteren Entscheidungen: Kommt die Biene an eine Blüte, muss sie entscheiden, ob dies eine Linde ist, um zu wissen, ob sie auf der Blüte landen soll oder nicht. Das tut sie anhand der erkannten Merkmale der Blüte: Aussehen und Duft. Derartige Entscheidungen können wir experimentell untersuchen. Wir können eine künstliche Wiese schaffen und beobachten, wie lange eine Biene braucht, um eine bestimmte Blüte zu besuchen oder um die Blüte abzulehnen.

Aus solchen Versuchen an frei fliegenden Bienen haben wir gelernt, dass die Zeit, die die Biene für eine Entscheidung benötigt, nicht von der Schwierigkeit der Aufgabe abhängt. Im Gehirn findet also für diese Entscheidung - weiterfliegen oder bleiben - ein bestimmter Ablauf statt, der nicht durch den Schwierigkeitsgrad der Duftunterscheidung beeinflusst wird.

Was hier passiert, können wir genauer untersuchen. Wir können unsere reduktionistische Schraube weiter andrehen, noch mehr Variablen ausschalten, dem Tier weniger Freiheitsgrade lassen. Damit schaffen wir Umstände, die zu besser reproduzierbaren Ergebnissen führen, damit wir einen aussagekräftigeren statistischen Zugang haben.

Bienen können im Experiment einen Blütenduft auch lernen, wenn sie nicht mehr fliegen. Dazu werden einzelne Bienen in Röhrchen festgehalten. Wenn man einer Biene einen Duft anbietet, reagiert sie

10 Kinder sind da meist anderer Meinung. 
nicht, wenn man aber den Bienenrüssel mit Zuckerwasser berührt, so löst dies den Rüsselreflex aus: Die Biene streckt den Rüssel aus und leckt am Zuckerwasser, das für die Biene wie Nektar ist. Wenn man nun einen Duft anbietet und gleich darauf das Zuckerwasser, so lernt die Biene, dass auf Duft Zuckerwasser folgt. Und das nächste Mal, wenn der Duft allein gegeben wird, streckt die Biene erwartungsvoll den Rüssel aus, selbst wenn gar kein Zuckerwasser gegeben wird. Dies ist eine klassische Konditionierung nach Pavlow. Grundlage für dieses Lernen ist ein Reflex, und daher würden wir in diesem Zusammenhang intuitiv nicht von einer freien Entscheidung reden. Die Biene hat gelernt, dass das Ereignis „Duft" das Ereignis „Zuckerwasser" vorhersagt. Sie reagiert auf den Duft damit, dass sie uns die Zunge entgegenstreckt.

Aber ganz so einfach ist das Bild nicht, denn die Bienen lernen nicht zu $100 \%$, sondern nur zu einem Teil. Wenn wir also 100 Bienen trainieren und dann alle testen, so wird nur ein Teil davon antworten, und zwar mit einer Quote von ca. 70\%. Was ist passiert? Entweder 70\% der Bienen haben gelernt und 30\% nicht. Vielleicht erlaubt es das Experiment, „dumme“ und ,intelligente“ Bienen zu unterscheiden, und tatsächlich macht dies einen Teil der Antwort aus, aber eben nur einen Teil. Die größte Komponente ist nämlich für den Beobachter zufällig. Wenn wir die $70 \%$ der Tiere, die auf den Duft reagiert haben, erneut testen, finden wir denselben Prozentsatz von Antworten wie zuvor. Ebenso, wenn man die restlichen 30\% einem erneuten Test unterzieht. Mit anderen Worten: Eine Lernquote von $70 \%$ bedeutet nicht, dass $70 \%$ der Tiere intelligenter sind und gelernt haben, sondern dass jedes Tier einzeln mit 70\% Wahrscheinlichkeit auf den Duft mit dem Rüsselreflex antworten wird. Von außen betrachtet hat also jede Biene Entscheidungsfreiheit, mit dem Zungenreflex zu reagieren - oder auch nicht. Hat die Biene einen freien Willen, trifft sie gar eine bewusste Entscheidung? Nicht nur die Antwort auf diese Frage entzieht sich unserem Zugang, sondern die Frage selbst bewegt sich außerhalb unserer Grenzen. Was wir aber sagen können ist, dass bei dieser Aufgabe die „Freiheit" klare statistische Grenzen hat. Wo diese Freiheit im Gehirn entsteht - also wodurch die statistische Verteilung zustande kommt - wissen wir noch nicht. Aber wir wissen schon einiges darüber, was dabei im Gehirn passiert.

Wir können die Aktivität der Neurone, die auf einen Duftreiz antworten, mit bildgebenden Verfahren messen. Die Aktivität in den für Düfte zuständigen Neuronen ist die Voraussetzung dafür, dass die Biene den Duft riecht. Diese Neurone sind Teil eines Schaltkreises von den 
Sinneszellen über die neuronalen Netzwerke im Gehirn zu den Muskeln der Zunge, die das Lecken ausführen. Was tun diese Zellen, wenn es keinen Duft gibt? Sind sie im schlafenden Zustand, still, bis zu einem neuen Dufterlebnis? Mitnichten! Wir können auch ohne einen Reiz zu präsentieren ihre Aktivität messen, und dann zeigt sich, dass ununterbrochen praktisch alle Neurone aktiv sind. Bei solchen Messungen schauen wir dem Gehirn in seiner Aktivität zu. Wäre dies ein menschliches Gehirn, so würden wir sagen: Wir schauen dem Gehim beim Denken zu. Da wir nicht wissen, was „Denken“ für ein Tier bedeutet (außer wir definieren „Denken“ so restriktiv, dass es nicht mehr unserem Sprachgebrauch entspricht - wir haben es hier mit einer ähnlichen begrifflichen Unschärfe zu tun wie beim ,Bewusstsein“"), reden wir pragmatisch und etwas umpoetisch von "Spontanaktivität" oder „Hintergrundaktivität". Wir schauen also dem Gehim bei seiner Spontanaktivität zu. Ist diese Spontanaktivität zufälig oder ist sie geordnet? Können wir gar, wie Andreas Herz angeboten hat," in der Stochastizität solcher Spontanaktivitäten die Grundlage für die Unvorhersagbarkeit einzelner Handlungen finden, also die Grundlage fuir die Freiheit des Handelns? Wie gesagt, die Frage nach der Freiheit ist nicht beantwortbar. Aber die Frage nach der statistischen Zufalligkeit ist untersuchbar, und tatsächlich: Die Muster sind nicht zufallig. So finden wir, kurz nachdem die Biene einen Duft gerochen hat, dass sich die Folge der spontanen Muster stärker an dem gerochenen Duft orientiert. Wohlgemerkt: Die spontane Aktivität bleibt in jedem Augenblick für uns unvorhersagbar und chaotisch, aber sie ist mitnichten zufällig, sondern folgt beschreibbaren statistischen Gesetzen. Ebenso ist es nicht möglich vorherzusagen, ob die einzelne Biene, nachdem sie konditioniert wurde, den Rüssel herausstreckt, obwohl wir die Wahrscheinlichkeit, mit der sie den Rüssel herausstreckt, benennen können.

Müssen wir also vor der Statistik kapitulieren oder können wir uns doch tiefer in die mechanistischen Zusammenhänge hineinwagen? Ich möchte letzteres anhand eines Beispiels versuchen. Ich habe schon erwähnt, dass Bienen im Stock viele Aufgaben übernehmen müssen. Das Leben einer Biene ist wohl geordnet: Nach dem Schlüpfen sorgt sich die junge Biene um das Innenleben im Stock, putzt und pflegt die Brut. Später wird sie zur Wächterin, die am Stockeingang fremde Tiere fernhält, eine Aufgabe, die sie vornehmlich aufgrund des Geruchs lösen kann: Wer fremd riecht, gehört nicht dazu und wird abgestochen.

11 Vgl. den Beitrag von Andreas Herz in diesen Band. 
Schließlich wird sie zur Sammlerin, fliegt aus und bringt Nektar und Pollen mit nach Hause. Diese Stadien werden auch Kasten genannt.

Das geregelte Leben, bei dem das Alter die Kaste einer Biene bestimmt, lässt sich experimentell durcheinanderbringen. Wir können Bienenstöcke so manipulieren, dass alle Bienen gleich alt sind, z. B. alle jung und dem Alter nach im Innendienst. Das hat keine schwerwiegenden Folgen: In einem wunderbaren Prozess der Selbstorganisation ändern einige Bienen ihre Aufgaben und beginnen auszufliegen, um Nektar und Pollen zu sammeln. Wie entscheidet die einzelne Biene nun, was sie tun soll? Ist jede Biene gewissermaßen „frei“ in ihrer Entscheidung, ihre Kaste zu ändern oder beizubehalten?

Als Experimentalisten können wir nur nach denjenigen Mechanismen suchen, die zu einer Entscheidungsfindung beitragen. Der Beitrag dieser Mechanismen reduziert den Anteil, der von außen als „frei“ betrachtet werden kann, da er nicht erklärt ist. Dieses Verbleibende ist Zufall, Varianz oder Freiheit - und selbst wenn wir diesen Teil statistisch über viele Tiere hinweg beschreiben können, bleibt die Entscheidung des Einzelnen immer eine Überraschung und somit nicht erklärbar. Wie bei der Frage der individuellen Handlung entzieht sich also der Beweis der Freiheit grundsätzlich dem Instrumentarium der empirischen Wissenschaft.

Bei den Bienen kennen wir einige der Faktoren, die für das einzelne Tier bei der Entscheidung auszufliegen oder im Innendienst zu bleiben eine Rolle spielen. Nehmen Sie die experimentelle Situation, in der alle Bienen gleich alt sind, das Alter also kein solcher Faktor ist. Unter solchen Bedingungen lassen sich andere Faktoren untersuchen. Es gibt ein Gen - das forager Gen - das hier eine große Rolle spielt: Jedes Tier exprimiert dieses Gen, aber nicht alle in gleichem Maße. Tiere, die dieses Gen stark exprimieren, neigen dazu, den Stock zu verlassen, während die, die weniger davon exprimieren, eher im Inneren bleiben. Man könnte sagen, das Gen regelt das Verhalten - aber auch dieses Gen funktioniert nicht allein, sondern im Netzwerk mit anderen Genen und vor allem im Zusammenspiel mit den anderen Individuen im Stock, denn die Schwelle, die zu einer Entscheidung „Ausfliegen“ führt, ist nicht durch das Gen selbst gegeben, sondern durch die Futterversorgung im Stock sowie durch die Anzahl anderer Individuen, die schon im Hausdienst tätig sind. Es ist etwa so wie in einer Wohngemeinschaft: Die Schwelle, endlich die Teller zu waschen (auch das ist eine Verhaltensentscheidung, mal mehr, mal weniger bewusst, mal mehr, mal weniger frei) ist bei jedem unterschiedlich und hängt von der Tages- 
form, dem Geruch in der Küche, der Müdigkeit und vielem mehr ab. Und doch: Es sind immer dieselben, deren Schwelle zum Abspülen als erste überschritten wird.

Was ist dieses forager Gen, das die Entscheidungsschwelle bei Bienen beeinflusst? Es kodiert ein ganz bestimmtes Enzym (eine cGMP-abhängige Proteinkinase). Bei Bienen steuert dieses Gen die Lichtempfindlichkeit - und zwar nicht in den Sinneszellen, sondern im Gehim. Lichtempfindlichere Bienen bleiben lieber im Stock und erledigen den Innendienst, während es die, bei denen das forager Gen stärker aktiv ist, eher nach außen ans Licht zieht: Sie werden zu Sammlerinnen. Ein ganz einfaches System, das in der Selbstorganisation des Stocks eine große Wirkung zeigt.

Dieses Gen ist kein besonderes Bienengen, auch bei Fruchtfliegen führt es zu unterschiedlichen Entscheidungen in der Futtersuche: Die Fliegenlarven, die forager stärker exprimieren, wandern bei der Futtersuche stärker umher als die Tiere, deren Aktivität in diesem Gen geringer ist. Hier haben wir also ein einzelnes Gen, das eine wichtige Stellschraube im Entscheidungsprozess eines Tieres darstellt. Solche Gene haben auch Menschen, und sie beeinflussen bei uns die Schwellen, nach denen wir unser Verhalten steuern. Welches Gen an den Schwellen für das Tellerwaschen in Wohngemeinschaften beteiligt ist, muss noch untersucht werden. Es könnte sich durchaus um ein Gen handeln, das die Geruchsempfindlichkeit beeinflusst und ganz ähnlich wie fortger wirkt.

\section{Bewusstsein ist weder in einem Zeitpunkt noch in einem Ort definierbar}

Ich habe bei den Begriffen „Bewusstsein“ und „Freiheit" darauf hingewiesen, dass diese schlecht definiert sind und sie sich deswegen der neurowissenschaftlichen Analyse entziehen. Die Problematik entsteht auch dadurch, dass sich beide Begriffe im allgemeinen Verständnis als etwas sehr Konkretes darstellen: Ich bin mir bewusst, dass ich frei handle. Die Ich-Person und das Jetzt sind klare Bestandteile unserer Vorstellung des Bewusstseins, und so wundert es nicht, dass mit Descartes einer der einflussreichsten Denker des Abendlandes sich bei der Frage nach der Ortung des Bewusstseins im Gehirn einen konkreten Ort dachte - das Pinealorgan. Sicher hat er die Rolle des Bewusstseins immer in der 
Gegenwart gesehen - in der Gegenwart des "Jetzt“, also zu einem bestimmten Zeitpunkt. Für Descartes gibt es einen Homunkulus im Gehirn, der das Bewusstsein darstellt. Dieses Bild steht klar im Widerspruch zur heutigen neurobiologischen Forschung: Wenn es ein $\mathrm{Be}-$ wusstsein gibt, egal wie es geartet sein mag, dann ist es weder an einem Ort, noch an einem Zeitpunkt festzumachen. ${ }^{12}$

Ich will dies an einem Beispiel verdeutlichen: Was passiert, wenn wir etwas bewusst und laut lesen? Prima facie beschreibt das Wort „lesen“ das, was wir tun. Uns ist genau in dem Moment, in dem wir einen Satz lesen, der Inhalt dieses Satzes, die Bedeutung der einzelnen Wörter, der Zusammenhang der verschiedenen Sätze bewusst. Aber so einfach ist es nicht: Wenn wir uns genau anschauen, was im Gehirn passiert, stellen wir fest, dass verschiedene Vorgänge, die in unserem Bewusstsein gleichzeitig ablaufen, in Wirklichkeit zeitversetzt sind, ohne dass uns das einschränkt.

Wir sehen die Buchstaben mit den Augen. Die optische Information gelangt aus der Netzhaut zuerst in den Thalamus und daraufhin in den visuellen Kortex, was über 40 ms dauert und mindestens vier Synapsen weit entfernt ist. Dann werden die Bilder in zwei parallel verlaufenden Kaskaden im Gehirn verarbeitet. Schließlich formen die Sprachzentren die Wörter, das motorische Zentrum kontrolliert - unter Einbezug eines seitlichen Abzweigs im Kleinhirn - die Muskelbewegungen in Mund und Rachen, und wir sprechen aus, was wir gelesen haben. Das, was wir sprechen, ist nicht gleichzeitig mit dem, was wir sehen. Aber hier hört der Vorgang nicht auf. Das ausgesprochene Wort wird vom Ohr wahrgenommen. Wieder findet eine Kaskade von neuronalen Ereignissen statt: Aus den Sinneszellen im Ohr über mehrere Schaltstellen bis zum akustischen Zentrum. Die Information vom akustischen System, die identisch sein muss mit der aus dem optischen System, kommt in Wirklichkeit erst viel später in gemeinsamen Gehirnarealen an - und doch haben wir nicht das Gefuihl, dass wir wie in einem Echo alles zwei oder gar dreimal durchmachen: sehen, sprechen, hören. Unser bewusster Eindruck ist, dass alles gleichzeitig stattfindet. Man könnte sich vorstellen, dass wir nur einen dieser Eingänge wirklich verarbeiten, doch das ist nicht der Fall: Die Verzögerung ist feststehend, ähnlich wie im oben erwähnten Beispiel, bei dem die vor Blüten flie-

12 Michael Stadler hat in seinem Beitrag in diesem Band darauf hingewiesen, dass die psychologische „Gegenwart" kein Zeitpunkt ist, sondern ein Zeitraum, der mehrere Sekunden lang dauert. 
genden Bienen für ihre Entscheidung, entweder zu landen oder nicht zu landen, ein festes Zeitintervall benötigen. Wenn wir diese zeitliche Verzögerung manipulieren - also wenn wir die Ungleichheit stören dann gerät das ganze System durcheinander. Dies lässt sich experimentell leicht zeigen: In einem solchen Experiment liest jemand einen Text in ein Mikrophon und hört seine eigene Stimme in nach außen isolierten Kopfhörern. Normalerweise kann man unter solchen Bedingungen sehr gut vorlesen. Wenn die Stimme aber elektronisch verzögert kommt, z.B. um $200 \mathrm{~ms}$, so gerät der Leser ins Stottern und der Lesefluss wird langsamer: Die zu falscher Zeit erfolgende akustische Rückkopplung bringt den Leser durcheinander. Dieses Phänomen ist unter der Bezeichnung DAF für ",delayed auditory feedback" bekannt. Es wird in der Sprachforschung eingesetzt, unter anderem in der Stottertherapie. Es zeigt aber auch, dass unser bewusstes „Ich“ im Gehirn nicht in einem einzigen Zeitpunkt zu verorten ist: Unser Gehirn funktioniert nicht wie ein Computer, der sequenziell arbeitet. Es gibt kein Jetzt, sondern das Jetzt ist ein - neuronal gesehen - ausgedehnter Zeitraum. ${ }^{1.3}$

Aus der Innenperspektive bleibt das Bewusstsein ein Zeitpunkt, der notwendigerweise am Ende aller Gehirnaktivitäten, die mit der Handlung zusammenhängen, stehen muss. Wenn Sie nach einem Glas greifen, muss das bewusste Gefühl mit der Handlung und nicht mit der Entscheidung zu handeln zusammenfallen, sonst würden Sie, wie beim DAF, Entscheidung und Handlung zeitversetzt erleben und ins „Handlungsstottern“ kommen. Die Gehirnströme, die am ehesten mit "Bewusstsein" korrelieren, können also nur nach denen kommen, die am ehesten mit „Entscheidungsfindung“ korrelieren. Dies wird von manchen dann so beschrieben, als sei das Bewusstsein eine aufgesetzte Illusion. Wenn wir aber das Bewusstsein aus seinem Zeitpunkt befreien und verstehen, dass es sich um eine Zeitspanne handelt, dann löst sich der Widerspruch auf. Analog zur Relativität der Zeit in der physikalischen Welt muss auch der Zeitbegriff für das Bewusstsein relativiert werden.

13 Darum ist das Libet-Experiment in seinen Grundannahmen falsch, demn es macht überhaupt keinen Simn, die Entscheidungsfindung in einen Zeitpumkt quetschen zu wollen. Allerdings ist aus diesem Fehler der logische Schluss unzulässig, dass die Schlussfolgerungen des Libet Experiments auch falsch sein müssen. Wichtig ist: Selbst wenn wir individuell das Gefuihl haben, dass wir uns unserer selbst immer zum jetzigen Zeitpunkt bewusst sind, so ist das ein Irrtum. Für das Gehim ist ,Jetzt" eine Zeitspanne und kein Zeitpunkt. 
Ähnlich wie es keinen Zeitpunkt für das Bewusstsein gibt, ist es mit dem Ort des Bewusstseins: Es hat keinen Ort; aus grundsätzlichen Erwägungen heraus kann es keinen Ort für das Bewusstsein geben. Wenn aber das Bewusstsein eine Netzwerkeigenschaft ist, die sich über die neuronalen Netzwerke des Gehirns sowohl räumlich als auch zeitlich erstreckt, dann bedeutet das, dass es nicht sinnvoll ist, die Funktion des „Bewusstseins“ des Gehirns getrennt von allen anderen Funktionen des Gehirns, ja selbst des gesamten Körpers zu betrachten. Es hat einmal jemand gesagt, er wisse nicht, wie viele Kriege ihre Ursache in einer Magenverstimmung hätten. Mit anderen Worten: Die Peristaltikbewegung des Darmes ist genauso ein Bestandteil im Entscheidungsprozess einer Handlung wie unser Gefuihl des Bewusstseins.

\section{Zusammenfassung und Schlussbemerkung}

Ich denke, wir haben einen freien Willen. Ich formuliere bewusst ,ich denke" und nicht ,ich weiß", denn dies ist kein empirischer Befund. Das Bewusstsein, so wie auch Moral und Ästhetik, haben sich in der Evolution durchgesetzt, um bessere Entscheidungen im sozialen Kontext zu ermöglichen. Aber diese Aussage treffe ich nicht als Neurowissenschaftler, sondern als denkender Mensch. Selbst die Evolutionsbiologie kann diese Aussage nicht belegen, denn um einen kausalen oder auch nur korrelativen Zusammenhang nahezulegen, bräuchten wir mehrere Arten, die wir vergleichen könnten. Doch Bewusstsein kennen wir bisher nur beim Menschen.

Aus neurobiologischer Sicht lässt sich jedoch klar sagen, dass die Willensfreiheit, die wir haben, nicht der freie Wille unseres bewussten Ichs ist - denn solch ein Bewusstsein, das wie ein Homunkulus als eigene Entität zu betrachten wäre, kann es nicht geben. Es ist der freie Wille von uns als ganzer Person. Forschungsgegenstand und Aufgabe der Psychologie ist es zu untersuchen, wie wir - als einzelne Menschen - in bestimmten Situationen bestimmte Entscheidungen treffen. Wie wir als Gruppen Entscheidungen treffen, wird von der Soziologie untersucht. Die Konsequenzen dieser Einsichten für unser Denken über uns selbst aufzuspüren, ist Aufgabe der Philosophie. Wie das Gehirn das tut, das können die Neurowissenschaften eruieren - aber nicht begründen, dass das Gehirn es tut. Damit wir über die Disziplinen hinweg unsere Grenzen sehen und uns trotzdem über diese Grenzen hinweg verstehen, benötigen wir den interdisziplinären Diskurs. 
- Die Neurowissenschaften sind eine experimentelle und empirische Wissenschaft - wenn sie diesen Boden verlassen, werden sie zu einer anderen Disziplin, etwa der Neurophilosophie, und es gelten andere Regeln.

- Tiere treffen - genauso wie wir Menschen - Entscheidungen, und in manchen Fällen kennen wir die rückgekoppelten Entscheidungsnetzwerke, zu denen Gene, Gehime und Gemeinschaften beitragen.

- Sowohl „Freiheit" als auch „Bewusstsein“ sind Begriffe, die innerhalb der Neurowissenschaften nicht sauber definierbar sind und sich dem Instrumentarium der Neurowissenschaften entziehen.

- Wir können aber beweisen, dass Bewusstsein weder an einem Ort noch an einer Zeit festzumachen ist. Deswegen muss man anders über Bewusstsein nachdenken, als es üblicherweise der Fall ist.

\section{Bibliographic}

Ben-Shahar, Yehuda (2005): The Foraging Gene, Behavioral Plasticity, and Honeybee Division of Labor. In: Journal of Comparative Pliysiology' A: Neuroethology, Sensory, Neural, and Behavioral Physiology (191), 987-994.

Ditzen, Mathias/Evers, Jan-Felix/Galizia, C. Giovanni (2003): Odor Similarity Does not Influence the Time Needed for Odor Processing. In: Chemical senses (28), $781-789$.

Galizia, C. Giovanni/Menzel, Randolf (2001): The Role of Glomeruli in the Neural Representation of Odours: Results from Optical Recording Studies. In: Jommal of Insect Physiology (47), 115-130.

Gerhardt, Volker/Heilinger, Jan-Christoph (2005): Funktionen des Bewusstseins. Antrag beim Bundesministerium für Bildung und Forschung zur Einrichtung einer Forschergruppe (Ms.).

Howell, Peter/Archer, Alexander (1984): Susceptibility to the Effects of Delayed Auditory Feedback. In: Perception \& Psychophysics (36), 296-302.

Jones-Smith, Katherine/Mathur, Harsh (2006): Fractal Analysis: Revisiting Pollock's Drip Paintings. In: Nature (444), E9-10; discussion E10-11.

Menzel, Randolf/Giurfa, Martin (2001): Cognitive Architecture of a MiniBrain: The Honeybee. In: Trends in Cognitive Sciences (5), 62-71.

Menzel, Randolf/Müller, Uli (1996): Learning and Memory in Honeybees: From Behavior to Neural Substrates. In: Anmual Revicw of Neuroscience (19), $379-404$.

Taylor, Richard P./Micolich, Adam P./David, Jonas (1999): Fractal Analysis of Pollock's Drip Paintings. In: Nature (399), 422.

Taylor, Richard P./Micolich, Adam P./Jonas, David (2006): Fractal Analysis: Revisiting Pollock's Drip Paintings (Reply). In: Nature (444), E10-11.

Wittgenstein, Ludwig (1963): Tractatus logico-philosophicus. Frankfurt am Main: Suhrkamp. 